\title{
Discerning Bulk and Interfacial Polarons in a Dual Electron Donor/ Acceptor Polymer
}

\author{
Jose M. Marin-Beloqui, ${ }^{\dagger}$ Kealan J. Fallon, ${ }^{\ddagger}$ Hugo Bronstein, ${ }^{\ddagger \odot}$ and Tracey M. Clarke, ${ }^{* \dagger}$ \\ ${ }^{\dagger}$ Department of Chemistry, University College London, Christopher Ingold Building, London WC1H 0AJ, United Kingdom \\ ${ }^{\ddagger}$ Department of Chemistry, University of Cambridge, Lensfield Road, Cambridge CB2 1EW, United Kingdom
}

\section{Supporting Information}

\begin{abstract}
The active layer of organic solar cells typically possesses a complex morphology, with amorphous donor/acceptor mixed domains present in addition to purer, more crystalline domains. These crystalline domains may represent an energy sink for free charges that aids charge separation and suppresses bimolecular recombination. The first step in exploiting this behavior is the identification and characterization of charges located in these different domains. Herein, the generation and recombination of both bulk and interfacial polarons are demonstrated in the dual electron donor/acceptor polymer XIND using transient absorption spectroscopy. The absorption spectra of XIND bulk polarons, present in pristine polymer domains, are clearly distinguishable from those of polarons present at the donor/acceptor interface. Furthermore, it is shown that photogenerated polarons are transferred from the interface to the bulk. These findings support the energy sink hypothesis and offer a way to maximize morphology relationships to enhance charge generation and suppress recombination.
\end{abstract}

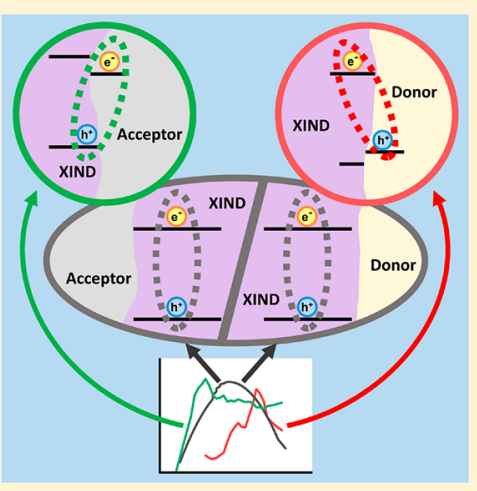

$\mathrm{D}$ espite organic photovoltaic (OPV) devices recently exhibiting high power conversion efficiencies $\left(>15 \%^{1-4}\right)$, they are still quite far from the theoretical limit efficiency of $23-24 \% .^{5,6}$ This disparity depicts the need for more fundamental studies of the different processes that occur at the donor/acceptor interface. Bimolecular recombination of free charges before they reach their respective electrodes has been established as one of the main loss mechanisms in OPV devices. ${ }^{7-10}$ It has been proposed that bimolecular recombination can be inhibited in active layers with a morphology comprised of both pure, crystalline domains and amorphous/ mixed domains. The crystalline domains would have a greater electron affinity in the case of the acceptor and/or a smaller ionization potential for donors, thereby creating energy sinks for charge carriers. ${ }^{11,12}$ In essence, this creates not only an additional driving force for charge separation but also an energetic barrier for bimolecular recombination. ${ }^{11,13}$ Both traits are highly desirable in OPV devices, and thus, control of morphology to achieve such energy sinks is critical. To do so, it is vital to characterize the polarons formed in such complex morphologies.

Transient absorption spectroscopy (TAS) is a useful technique for measuring bimolecular recombination, as the polaron signal amplitude is directly proportional to the number of charges generated in the blend. ${ }^{14}$ The TAS polaron signal amplitude at $1 \mu \mathrm{s}$ has been shown to be directly proportional to the short circuit current, $J_{\mathrm{sc}}$ of an operational photovoltaic device. ${ }^{15}$ In addition, it has recently been shown that there is a clear correlation between the polaron TA intensity and the external quantum efficiency (EQE) of a device. ${ }^{16}$
Herein, we study a novel cross-conjugated polymer, XIND (Figure 1a, full name available in the Supporting Information). ${ }^{17}$ As there is no pathway of conjugation across the IND core (in the area circled in Figure 1a), the effective conjugation length of this polymer is decreased, leading to a wider optical band gap, higher ionization potentials, and greater oxidative stability compared to those of its fully conjugated counterpart. Cross-conjugation leads to a localization of the $\mathrm{HOMO}$ and partial charge transfer (CT) character of the primary HOMOLUMO transition. ${ }^{17}$ XIND may therefore be capable of acting as either an electron donor or electron acceptor [as also indicated by its HOMO and LUMO energy levels (Figure 1b)], thus allowing formation of negative and positive polymer polarons. This ambipolar character thus offers the ideal opportunity to explore the energy sink hypothesis. The dual ability of XIND to act as an electron donor (when blended with fullerenes $\mathrm{PC}_{60} \mathrm{BM}$ and $\mathrm{PC}_{70} \mathrm{BM}$ ) and as an electron acceptor (blended with $\mathrm{P} 3 \mathrm{HT}$ ) was demonstrated using microsecond TAS. Crucially, this dual character enabled the identification of interfacial negative and positive XIND polarons, demonstrating their completely different spectral signatures. Importantly, P3HT blends utilizing the XIND polymers as acceptors generated very similar P3HT polaron populations compared to those of standard fullerene blends, enabling the clear identification of the negative XIND polaron. Furthermore, spectral evolution and changes in decay dynamics over the microsecond time scale revealed the

Received: May 3, 2019

Accepted: June 19, 2019

Published: June 19, 2019 
a)

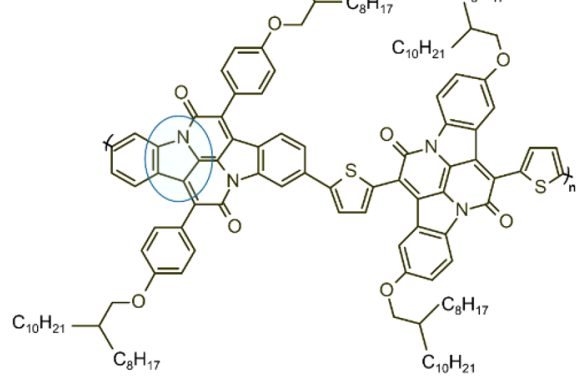

b)

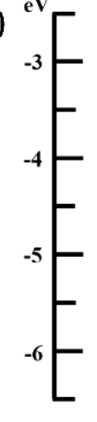

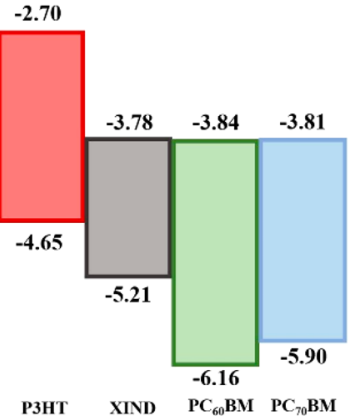

c)

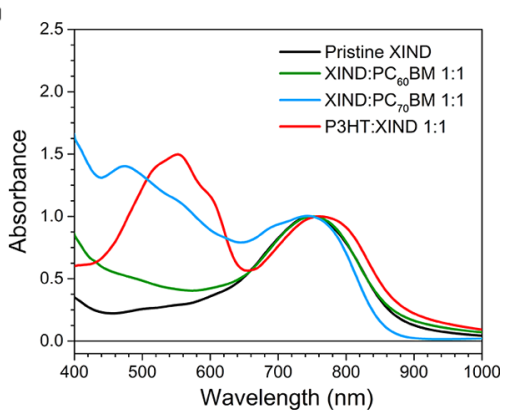

Figure 1. (a) Chemical structure of the cross-conjugated XIND polymer. The blue oval indicates where the conjugation along the polymer backbone is interrupted. (b) Energy level diagram for P3HT, XIND, PC60BM, and PC70BM. All HOMO values were taken from photoelectron spectroscopy results in the literature. LUMO values were estimated by adding the optical band gap. ${ }^{17-19}$ (c) Normalized ground state absorbance of a pristine XIND film (black) and films blended with $\mathrm{PC}_{60} \mathrm{BM}$ (green), $\mathrm{PC}_{70} \mathrm{BM}$ (blue), and $\mathrm{P} 3 \mathrm{HT}$ (red).

presence of both bulk and interfacial polarons, again possessing very different spectral signatures.

The ground state absorbance spectra for the pristine and blend XIND films studied in this work are shown in Figure 1c: pristine XIND, 1:1 XIND:PC ${ }_{60} \mathrm{BM}, 1: 1 \mathrm{XIND}: \mathrm{PC}_{70} \mathrm{BM}$, and 1:1 P3HT:XIND. Pristine XIND presents an absorption spectrum that extends into the near infrared (NIR) with a peak maximum centered at $\sim 750 \mathrm{~nm}$. The photoluminescence of XIND was very low; hence, quenching measurements could not be accurately used to assess exciton quenching (Figure S1).

Normalized (per photon absorbed) microsecond transient absorption spectra for these films are shown in Figure 2a. The excitation wavelength of $700 \mathrm{~nm}$ was chosen to selectively excite the XIND polymer. An excitation energy of $30 \mu \mathrm{J} / \mathrm{cm}^{2}$ was chosen to perform TAS as it was in the linear regime of charges generated and light absorbed (Figure S2). The pristine XIND TA spectrum shows a broad absorption centered at approximately $1350 \mathrm{~nm}$. This band was assigned to charge carriers, due to the lack of oxygen dependence (see Figure S3), suggesting an absence of triplet states. However, because the $S_{1}$ energy of XIND is at approximately $1.4 \mathrm{eV}$, then it is possible that the XIND $\mathrm{T}_{1}$ state is $<0.98 \mathrm{eV}$, the threshold for observing oxygen sensitivity. As such, the possibility that the $1350 \mathrm{~nm}$ band in pristine XIND is related to triplets rather than charges was explored in more detail. As shown in Figure $2 \mathrm{~b}$ and Figure S4, TA spectral evolution of XIND in $o$-dichlorobenzene solution shows two components: an oxygen-sensitive, slowerdecaying band at $1000 \mathrm{~nm}$ and a component at approximately $1350 \mathrm{~nm}$ with faster decay dynamics and a lack of oxygen sensitivity. Given that the ground state absorption maximum does not shift from film to solution, we can conclude that the $1350 \mathrm{~nm}$ band observed in both film and solution is the polaron while the $1000 \mathrm{~nm}$ band observed in the solution can be assigned to the triplet. This was confirmed via sensitization experiments in solution with the platinum porphyrin PtOEP, where the sensitized XIND triplet was observed at $1100 \mathrm{~nm}$ (Figure S5). The assignment of charges in the pristine XIND film implies that the broad $1350 \mathrm{~nm}$ band must possess both negative and positive polaron signatures.

When XIND was used as an electron donor, blended with $\mathrm{PC}_{60} \mathrm{BM}$, the TA spectrum measured at $1 \mu \mathrm{s}$ showed features similar to, but more well-defined than, those seen in the pristine polymer (Figure 2a). The TA amplitude of the blend, and thus the polaron population, is five times higher than that of the pristine sample, an extraordinary observation considering the almost zero LUMO level offset implies virtually no
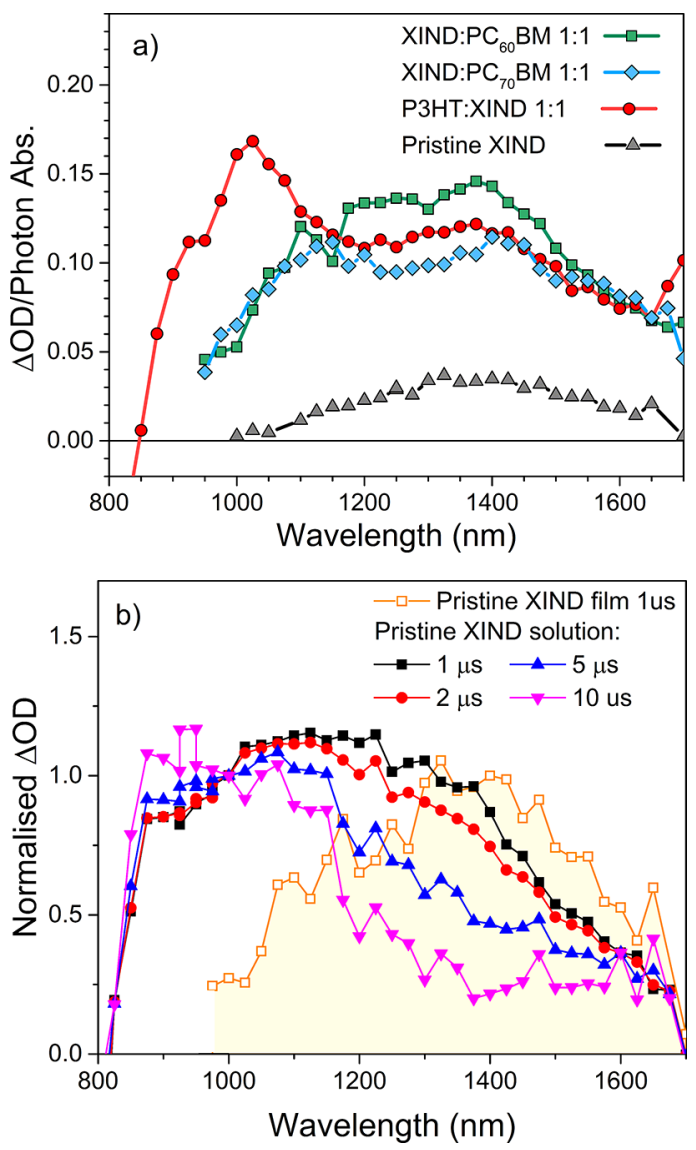

Figure 2. (a) Transient absorption spectra of films obtained at $1 \mu \mathrm{s}$ with an excitation density of $30 \mu \mathrm{J} / \mathrm{cm}^{2}$ for pristine XIND (gray triangles), XIND: $\mathrm{PC}_{60} \mathrm{BM}$ (green squares), XIND:PC ${ }_{70} \mathrm{BM}$ (blue diamonds), and P3HT:XIND (red circles) upon excitation at $700 \mathrm{~nm}$. Spectra have been normalized per photon absorbed. (b) The spectral evolution of a deaerated pristine XIND solution $\left(100 \mu \mathrm{g} \mathrm{mL}^{-1}\right.$ in $o$ dichlorobenzene), as compared to the $1 \mu \mathrm{s}$ TA spectrum of the pristine XIND film.

driving force for charge separation (when exciting the XIND). This has been observed for the narrower band gap conjugated backbone IND polymers as well, ${ }^{20}$ and suggests contributions from other factors, such as entropy, ${ }^{21}$ or strong coupling between the $S_{1}$ and charge transfer states. ${ }^{22}$ The XIND:PC ${ }_{70} \mathrm{BM}$ film presents features similar to those of its $\mathrm{PC}_{60} \mathrm{BM}$ counterpart (Figure $2 \mathrm{a}$ ), suggesting that all the 
spectral features observed relate to the polymer and not to fullerene anions. ${ }^{23,24}$

The P3HT:XIND transient spectrum at $1 \mu \mathrm{s}$ (Figure 2a), where XIND acts as electron acceptor, showed the expected peak around $1000 \mathrm{~nm}$ assigned to the $\mathrm{P} 3 \mathrm{HT}$ positive polaron. ${ }^{25}$ The additional band at $1400 \mathrm{~nm}$ must therefore correspond to XIND, consistent with the similarity of this feature to that seen in the pristine XIND polymer sample. The presence of the P3HT positive polaron, after sole excitation of the XIND polymer at $700 \mathrm{~nm}$, indicates efficient hole transfer from XIND to P3HT.

The similarity of the XIND TA spectra at $1 \mu \mathrm{s}$, regardless of whether it is acting as an electron donor or an electron acceptor, was unexpected, and thus the polaron decay dynamics were studied in more detail. Figure 3 shows the

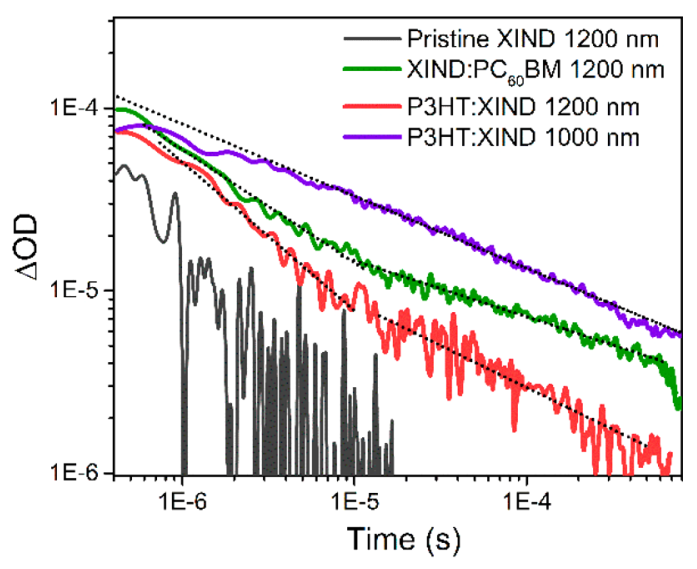

Figure 3. TA decay with excitation at $700 \mathrm{~nm}$ and an excitation density of $30 \mu \mathrm{J} / \mathrm{cm}^{2}$ for pristine XIND (black line) and XIND:PC ${ }_{60} \mathrm{BM}$ (green) probed at $1200 \mathrm{~nm}$, and P3HT:XIND probed at $1000 \mathrm{~nm}$ (purple) and $1200 \mathrm{~nm}$ (red). The black dotted lines represent the power law fittings. All blend samples are 1:1 by weight.

transient decay kinetics of the XIND films using a 1:1 blend ratio (XIND:PC ${ }_{70} \mathrm{BM}$ shown in Figure $\mathrm{S} 6$ due to its similarity to the $\mathrm{PC}_{60} \mathrm{BM}$ blend). The TA of the pristine XIND polymer charges decayed within a few microseconds. Despite this, the high initial $\Delta \mathrm{OD}$ indicates a relatively large number of charges are generated in the pristine material. This is quite rare behavior; only a few polymers have shown significant charge density on the microsecond time scale in the pristine material. $^{26}$

When the compound was blended with P3HT, the dynamics were probed on two different wavelengths: $1000 \mathrm{~nm}$ (P3HT polaron) and $1200 \mathrm{~nm}$ (XIND polaron). The decay dynamics of P3HT:XIND with a probe wavelength of $1000 \mathrm{~nm}$ show a single power law decay $\left(\Delta \mathrm{OD} \propto t^{-\alpha}\right)$ from approximately $2 \mu \mathrm{s}$ onward with an $\alpha$ value of 0.4 . This power law behavior is associated with bimolecular recombination limited by the thermal activation of charges localized in an exponential density of traps. ${ }^{27}$ Because XIND charged species do not absorb at $1000 \mathrm{~nm}$, the only species being monitored is the P3HT positive polaron. As such, this $\alpha=0.4$ power law decay can be attributed to recombination between $\mathrm{P} 3 \mathrm{HT}$ positive polarons and XIND negative polarons [the deviation of the power law seen at early times seems to be caused by XIND polymer ground state bleaching (Figure S7)]. When probed at the XIND polaron at $1200 \mathrm{~nm}$, two decay phases were obtained: a fast component that extends until $10 \mu$ s and a slow one that reaches the millisecond time range. Both components showed power law kinetics with $\alpha$ values of 0.8 and 0.4 for the fast and slow phases, respectively. Noting the similarity in $\alpha$ between the $1000 \mathrm{~nm}$ kinetics of the P3HT polaron and this $1200 \mathrm{~nm}$ probe slow phase, we found the slow phase can therefore also be assigned to recombination between P3HT positive polarons and XIND negative polarons. The $1200 \mathrm{~nm}$ probe fast phase, however, is not present when probing at the $\mathrm{P} 3 \mathrm{HT}$ polaron at $1000 \mathrm{~nm}$, and this suggests that it can be attributed solely to XIND species, charge recombination between XIND positive and negative polarons within pure XIND domains. This assignment is in accordance with the time scales observed for the same recombination process seen in the pristine XIND polymer sample. This conclusion is also supported by the observation that directly exciting the XIND leads to a higher relative amplitude of this fast phase compared to that of P3HT excitation (Figure S8).

XIND:PC ${ }_{60} \mathrm{BM}$ dynamics were probed at $1200 \mathrm{~nm}$. The TA decay again showed two different components: one fast component from $<1$ to $10 \mu \mathrm{s}$ and a slower one that continues over $1 \mathrm{~ms}$. Both components can be fitted to a power law, with $\alpha$ values of approximately 0.7 and 0.3 for the fast and slow phase, respectively. The $\alpha$ value of the fast phase in this $\mathrm{PC}_{60} \mathrm{BM}$ blend is similar to that of the P3HT blend, indicating that the fast phase in both blends can be associated with recombination between positive and negative XIND polarons inside pure XIND domains. The slower component was thus
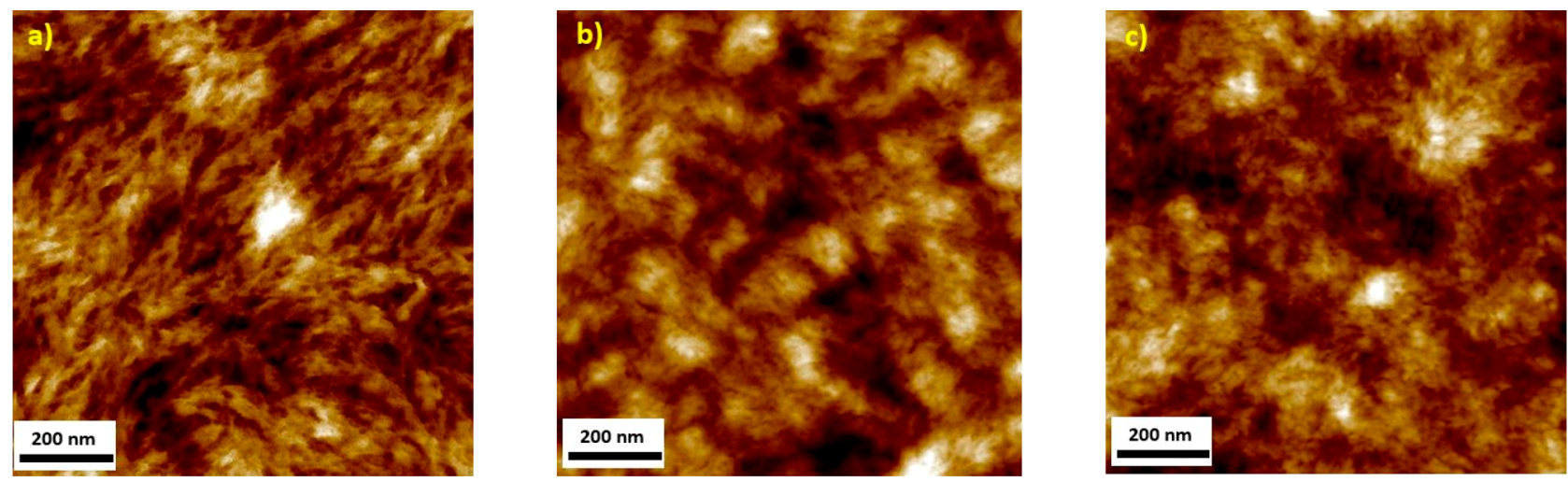

Figure 4. AFM images of (a) pristine XIND, (b) 1:1 XIND:PC ${ }_{60} \mathrm{BM}$, and (c) 1:1 P3HT:XIND films. Scale bars are $200 \mathrm{~nm}$. 

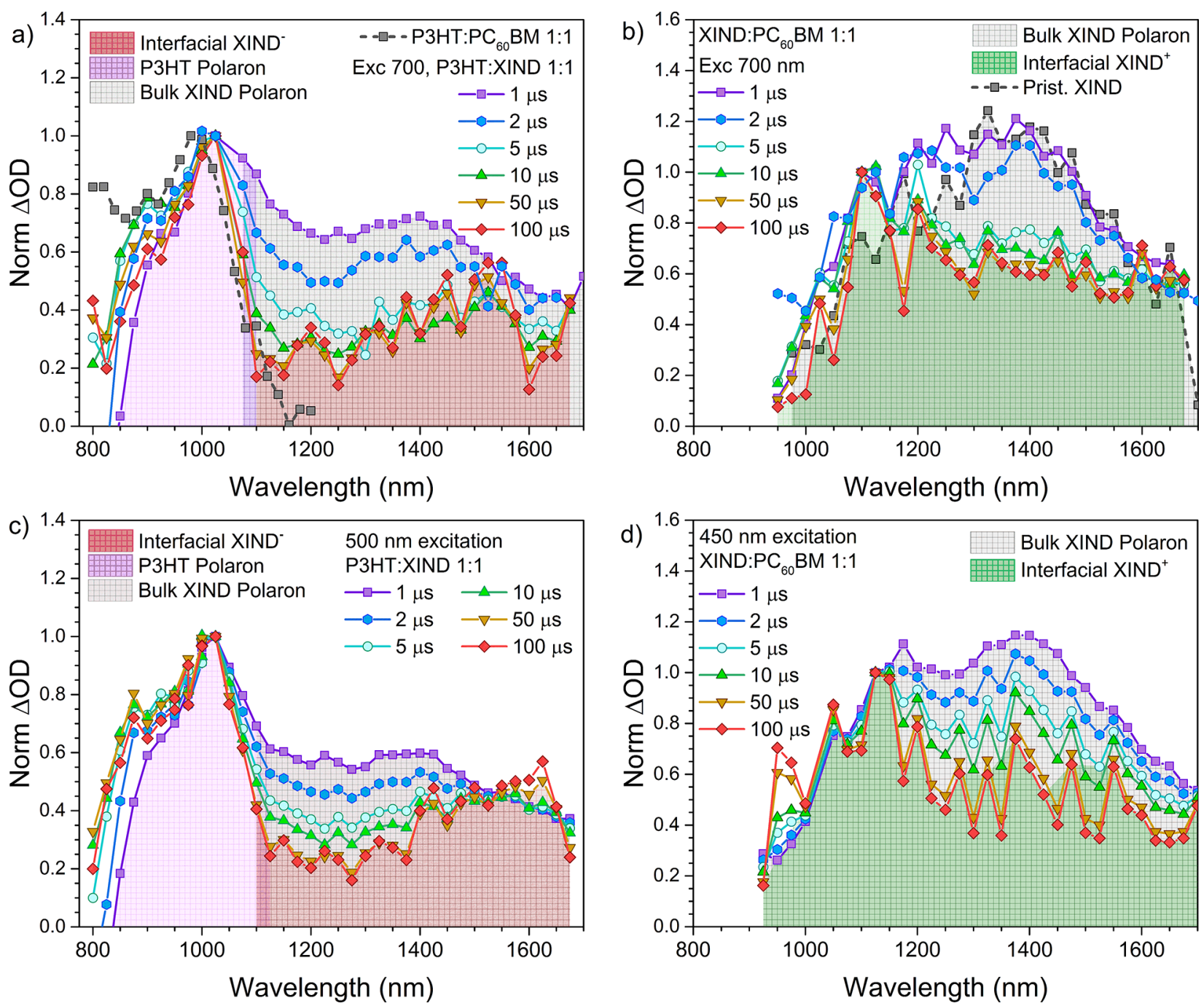

Figure 5. Normalized TA spectra recorded over a time range of 1-100 $\mu$ s of (a) P3HT:XIND and (b) XIND:PC ${ }_{60} \mathrm{BM}$ films, using 700 nm excitation, and (c) P3HT:XIND and (d) XIND:PC ${ }_{60} \mathrm{BM}$ films, using 500 and $450 \mathrm{~nm}$ excitation, respectively. P3HT:PCBM (black squares and dashed line) and pristine XIND TA spectra at $1 \mu$ s have been added to panels a and b, respectively, as references.

assigned to charge recombination between XIND positive polarons and PCBM anions. The $\alpha$ value obtained for this slow phase (0.3) is low compared with those of other $\mathrm{PC}_{60} \mathrm{BM}$ blends in the literature, ${ }^{28,29,27}$ implying the presence of deep trap states.

The excitation density dependence of the TA decay dynamics was examined for the XIND blends (Figure S9). Both XIND:PC ${ }_{60} \mathrm{BM}$ and P3HT:XIND behaved similarly, with no evident saturation even at high fluences. Comparing the excitation density dependence of the two decay phases at 1200 $\mathrm{nm}$ reveals virtually identical behavior, indicating that both recombination phases operate independently of one another.

The presence of these two polaron decay phases in the blends - an interfacial recombination between donor and acceptor and a bulk recombination inside pure XIND domains suggests the existence of large pure polymer domains in the blend samples. Atomic force microscopy (AFM) was used to verify this (Figure 4). As shown in Figure 4a, the pristine XIND film shows a fibril microstructure that is typically related to high crystallinity. ${ }^{30,31}$ Panels $\mathrm{b}$ and $\mathrm{c}$ of Figure 4 show the AFM images of 1:1 $\mathrm{PC}_{60} \mathrm{BM}$ and $\mathrm{P} 3 \mathrm{HT}$ blends, respectively. In both blends, the fibril morphology is lost and large domains $(\sim 90 \mathrm{~nm})$ are seen, as expected.
The presence of two recombination phases relating to bulk and interfacial polarons offers the opportunity to distinguish between these two polaron types spectrally, as well. The spectral evolution over time was therefore investigated, as shown in Figure 5. Figure 5a shows the P3HT:XIND spectral evolution normalized to the $\mathrm{P} 3 \mathrm{HT}$ positive polaron using 700 $\mathrm{nm}$ excitation. A clear spectral evolution is observed, with the broad $1400 \mathrm{~nm}$ band observed at $1 \mu$ s rapidly decaying to reveal a band at $1550 \mathrm{~nm}$ at longer times. The $1000 \mathrm{~nm}$ band, which does not shift over time, is identical to that of a P3HT:PCBM reference spectrum and thus remains assigned to the $\mathrm{P} 3 \mathrm{HT}$ positive polaron. The $1550 \mathrm{~nm}$ band, which is apparent on the time scales of the slow recombination phase between P3HT and XIND polarons, must therefore be assigned to interfacial negative XIND polarons. In contrast, the broad $1400 \mathrm{~nm}$ band that dominates at early times (and is very similar to that of the pristine XIND spectrum) is likely to be polarons formed within pure XIND domains (bulk polarons).

Figure $5 \mathrm{~b}$ shows the spectral evolution over time of the XIND: $\mathrm{PC}_{60} \mathrm{BM}$ blend using $700 \mathrm{~nm}$ excitation. A similar trend is observed, where the band centered at $1400 \mathrm{~nm}$ quickly decays within $10 \mu \mathrm{s}$. In this case, however, the absorption band 
at long times has a maximum around $1050 \mathrm{~nm}$. This feature was therefore assigned to the XIND positive polaron at the donor/acceptor interface. The same $1050 \mathrm{~nm}$ band is present after the $1400 \mathrm{~nm}$ band decays in the $\mathrm{PC}_{70} \mathrm{BM}$ blend (Figure S10), consistent with the assumption that this band belongs to the XIND positive polaron rather than a fullerene species. The negative fullerene anion is not apparent, likely due to its much lower absorptivity.

The similarity between the $1400 \mathrm{~nm}$ absorption in the XIND:PC ${ }_{60} \mathrm{BM}$ blend at $1 \mu \mathrm{s}$ and the pristine spectrum is highlighted in Figure 5b, supporting the assignment of this band to bulk polarons in pure XIND domains. Crucially, the pristine XIND TA shows no spectral evolution (Figure S11), indicating the presence of solely bulk polarons. Furthermore, the sum of the two individual components' spectra together, the bulk polaron spectrum (from the pristine XIND film) and the positive XIND interfacial polaron spectrum [from the $\mathrm{XIND}: \mathrm{PC}_{60} \mathrm{BM}$ blend recorded at $50 \mu \mathrm{s}$ (Figure S12)], is identical to that blend's spectrum at $1 \mu \mathrm{s}$. This clearly suggests that at early times both interfacial and bulk polarons are present simultaneously.

The observation of bulk polarons and spectrally distinct interfacial positive and negative XIND polarons was investigated further by varying the excitation wavelength. Altering the excitation wavelength to coincide with the second component of the blend [500 nm for P3HT:XIND and 450 $\mathrm{nm}$ for XIND:PC ${ }_{60} \mathrm{BM}$ (Figure $5 \mathrm{c}, \mathrm{d}$ )] resulted in behavior identical to that for XIND excitation, in terms of a rapid decay of XIND bulk polarons and long-lived interfacial polarons. Interestingly, despite the XIND component of the blend accounting for only $40 \%$ and $20 \%$ of the total light absorption for XIND:PC ${ }_{60} \mathrm{BM}$ and $\mathrm{P} 3 \mathrm{HT}$ :XIND, respectively, at these excitation wavelengths (Figure S13), the bulk XIND polaron amplitude (relative to the interfacial polaron) remains remarkably similar to that of selective XIND excitation at $700 \mathrm{~nm}$. In the case of the XIND:PC ${ }_{60} \mathrm{BM}$ blend, the bulk/ interfacial polaron ratio remains the same, while for the P3HT:XIND blend, it decreases by only $20 \%$. This observation implies that, despite the majority of excitons being photogenerated in the second blend component, the relative population of bulk XIND polarons at $1 \mu \mathrm{s}$ is similar to that observed for selective XIND photoexcitation. In conjunction with the prior observation that interfacial and bulk polarons are present simultaneously at $1 \mu \mathrm{s}$, the implication here is that a significant proportion of charges are transferred from the interface to the bulk at times prior to $1 \mu \mathrm{s}$.

The effect of the donor:acceptor ratio was also studied, with $1: 2$ and $1: 4$ ratios being tested in addition to the $1: 1$ ratio (Figure S13). For the P3HT:XIND blends, XIND excitation at $700 \mathrm{~nm}$ produces very similar polaron yields irrespective of the blend ratio and regardless of whether the interfacial P3HT polaron is probed $(1000 \mathrm{~nm})$ or the bulk XIND polaron (1200 $\mathrm{nm}$ ). In the case of the bulk XIND polaron, this is consistent with significant charge photogeneration occurring in the bulk of the XIND without any influence from P3HT. In the case of the interfacial polaron, it implies that hole transfer from XIND to P3HT is limited by accessibility to the interfacial area. For P3HT excitation at $500 \mathrm{~nm}$, both polaron yields change substantially across the blend ratio series, with the 1:1 ratio (the greatest interfacial area) producing the highest polaron yields. Crucially, the bulk XIND polaron population at 1400 $\mathrm{nm}$ increases from 1:4 to 1:1 with P3HT excitation, despite the decrease in relative ground state absorbance from the XIND component. As such, this is consistent with the hypothesis of charges being transferred from the interface into the bulk, with the efficiency of this process improving as the interfacial area is increased from $1: 4$ to $1: 1$.

These results are significant for numerous reasons. First, XIND positive and negative polarons are shown to absorb in different spectral regions (1050 and $1550 \mathrm{~nm}$, respectively). Typically negative and positive polymer polarons are expected to have identical absorption profiles, even in co-polymers. ${ }^{32}$ The large differences observed here are likely to be related to the cross-conjugated nature of XIND. Cross-conjugation essentially breaks the conjugation, inhibiting electronic coupling and allowing the two charged species to be spectrally distinct.

Second, the observation of both bulk and interfacial polarons simultaneously on the early microsecond time scales may be a general phenomenon and thus could affect spectral assignments in future donor/acceptor blends. Multiple polaron peaks have indeed been observed in other polymer/fullerene systems. ${ }^{28,33,34}$ Hypotheses for this behavior have typically centered around delocalized versus localized polarons within polymer domains, ${ }^{35}$ but these results show that the location of the charges (bulk or interfacial) may also play a significant role.

Indeed, the ability of XIND to act as both an electron donor and an electron acceptor has allowed bulk and interfacial polarons to be distinguished using TAS for the first time. The bulk polarons generated in pure XIND domains show a spectral profile completely different from those of the interfacial polarons observed at later times. The differing energetics implied by the energy sink hypothesis is consistent with the distinct spectral profiles of the bulk and interfacial polarons. It is important to note that the fast decay of the bulk polarons will likely have a large contribution from recombination of polarons photogenerated within the pure domain (not only polarons that have migrated there from the interfacial regions). In contrast, the remarkably slow decay of interfacial polarons (particularly for the XIND:PC ${ }_{60} \mathrm{BM}$ blend) may have a contribution from adjacent crystalline XIND domains acting as an energy sink, essentially acting as a trap for XIND polarons and inhibiting recombination. This is consistent with the excitation wavelength and blend ratio dependencies both suggesting a transfer of photogenerated charges from the interface to the bulk and thus provides support for the energy sink hypothesis.

XIND polarons originally generated at the interface that then undergo a long residence/trapping time in the crystalline domains should lead to a blurring of the interfacial and bulk XIND polaron spectra at long times, but this is not observed. There are a number of possible reasons for this. The presence of interfacial dipoles may enhance the molar absorptivity of the interfacial polarons over that of the bulk polarons, ${ }^{36}$ thus leading the interfacial polaron signal to dominate at longer times (when its concentration is higher than that of the bulk polaron). This is consistent with the asymmetry of the interfacial polaron peaks in both blends, suggesting the presence of a much weaker additional component that is obscured (the bulk polaron). Furthermore, trap (energy sink) sites for the interfacial XIND polarons may not be located as deep within the bulk as the initially generated bulk XIND polarons and thus could be in a different morphological environment. The extent of polaron delocalization may also play a role. 
In summary, a transient absorption spectroscopy study of the XIND polymer has shown its dual electron donor/acceptor character by the creation of XIND polarons when blended with either fullerene or P3HT. The consequent ability of the pristine XIND to generate charges in the absence of an acceptor was used to demonstrate both spectrally distinct negative and positive polarons and spectrally distinct bulk and interfacial polarons. The bulk and interfacial polarons not only displayed clearly different spectral features but also gave rise to different bimolecular recombination kinetics on the microsecond time scale. In particular, the interfacial polarons are unusually long-lived, possibly due to a residence time in lowenergy sinks created by crystalline XIND regions. This is also consistent with TA results as a function of excitation wavelength and blend ratio, which demonstrate a transfer of photogenerated polarons from the interface to the bulk, as suggested by the energy sink hypothesis. Finally, the observation of bulk and interfacial polarons provides an additional consideration for future spectral assignments.

\section{ASSOCIATED CONTENT}

\section{S Supporting Information}

The Supporting Information is available free of charge on the ACS Publications website at DOI: 10.1021/acs.jpclett.9b01264.

Experimental procedure, PL of XIND samples, oxygen sensitivity TA results, solution TA results, sensitization results, TA spectra normalized per photon absorbed, TA kinetics for all blend ratios and excitation wavelengths studied, TA saturation curves, and data for $\mathrm{PC}_{70} \mathrm{BM}$ blends (PDF)

\section{AUTHOR INFORMATION}

\section{Corresponding Author \\ *E-mail: tracey.clarke@ucl.ac.uk. \\ ORCID $\odot$}

Jose M. Marin-Beloqui: 0000-0003-1762-5595

Kealan J. Fallon: 0000-0001-6241-6034

Hugo Bronstein: 0000-0003-0293-8775

Tracey M. Clarke: 0000-0003-4943-0645

\section{Notes}

The authors declare no competing financial interest.

\section{ACKNOWLEDGMENTS}

T.M.C. acknowledges support from EPSRC Project EP/ N026411/1. H.B. acknowledges ERC Grant CONTREX.

\section{REFERENCES}

(1) Xiao, Z.; Jia, X.; Ding, L. Ternary Organic Solar Cells Offer 14\% Power Conversion Efficiency. Sci. Bull. 2017, 62, 1562-1564.

(2) Li, S.; Ye, L.; Zhao, W.; Yan, H.; Yang, B.; Liu, D.; Li, W.; Ade, H.; Hou, J. A Wide Band Gap Polymer with a Deep Highest Occupied Molecular Orbital Level Enables 14.2\% Efficiency in Polymer Solar Cells. J. Am. Chem. Soc. 2018, 140, 7159-7167.

(3) Zhang, S.; Qin, Y.; Zhu, J.; Hou, J. Over 14\% Efficiency in Polymer Solar Cells Enabled by a Chlorinated Polymer Donor. Adv. Mater. 2018, 30, 1800868.

(4) Yuan, J.; Zhang, Y.; Zhou, L.; Zhang, G.; Yip, H.-L.; Lau, T.-K.; Lu, X.; Zhu, C.; Peng, H.; Johnson, P. A.; et al. Single-Junction Organic Solar Cell with over 15\% Efficiency Using Fused-Ring Acceptor with Electron-Deficient Core. Joule 2019, 3, 1140-1151.
(5) Minnaert, B.; Burgelman, M. Efficiency Potential of Organic Bulk Heterojunction Solar Cells. Prog. Photovoltaics 2007, 15, 741748.

(6) Janssen, R. A. J.; Nelson, J. Factors Limiting Device Efficiency in Organic Photovoltaics. Adv. Mater. 2013, 25, 1847-1858.

(7) Kyaw, A. K. K.; Wang, D. H.; Gupta, V.; Leong, W. L.; Ke, L.; Bazan, G. C.; Heeger, A. J. Intensity Dependence of Current-Voltage Characteristics and Recombination in High-Efficiency SolutionProcessed Small-Molecule Solar Cells. ACS Nano 2013, 7, 45694577.

(8) Hawks, S. A.; Deledalle, F.; Yao, J.; Rebois, D. G.; Li, G.; Nelson, J.; Yang, Y.; Kirchartz, T.; Durrant, J. R. Relating Recombination, Density of States, and Device Performance in an Efficient Polymer:Fullerene Organic Solar Cell Blend. Adv. Energy Mater. 2013, 3, 1201-1209.

(9) Maurano, A.; Shuttle, C. G.; Hamilton, R.; Ballantyne, A. M.; Nelson, J.; Zhang, W.; Heeney, M.; Durrant, J. R. Transient Optoelectronic Analysis of Charge Carrier Losses in a Selenophene/Fullerene Blend Solar Cell. J. Phys. Chem. C 2011, 115, 59475957.

(10) Shuttle, C. G.; Hamilton, R.; O’Regan, B. C.; Nelson, J.; Durrant, J. R. Charge-Density-Based Analysis of the Current-Voltage Response of Polythiophene/Fullerene Photovoltaic Devices. Proc. Natl. Acad. Sci. U. S. A. 2010, 107, 16448-16452.

(11) Jamieson, F. C.; Domingo, E. B.; McCarthy-Ward, T.; Heeney, M.; Stingelin, N.; Durrant, J. R. Fullerene Crystallisation as a Key Driver of Charge Separation in Polymer/Fullerene Bulk Heterojunction Solar Cells. Chem. Sci. 2012, 3, 485-492.

(12) Clarke, T. M.; Lungenschmied, C.; Peet, J.; Drolet, N.; Mozer, A. J. Tuning Non-Langevin Recombination in an Organic Photovoltaic Blend Using a Processing Additive. J. Phys. Chem. C 2015, 119, 7016-7021.

(13) Tsoi, W. C.; Spencer, S. J.; Yang, L.; Ballantyne, A. M.; Nicholson, P. G.; Turnbull, A.; Shard, A. G.; Murphy, C. E.; Bradley, D. D. C.; Nelson, J.; et al. Effect of Crystallization on the Electronic Energy Levels and Thin Film Morphology of P3HT:PCBM Blends. Macromolecules 2011, 44, 2944-2952.

(14) Shuttle, C. G.; O’Regan, B.; Ballantyne, A. M.; Nelson, J.; Bradley, D. D. C.; Durrant, J. R. Bimolecular Recombination Losses in Polythiophene: Fullerene Solar Cells. Phys. Rev. B: Condens. Matter Mater. Phys. 2008, 78, 113201.

(15) Clarke, T. M.; Ballantyne, A.; Shoaee, S.; Soon, Y. W.; Duffy, W.; Heeney, M.; McCulloch, I.; Nelson, J.; Durrant, J. R. Analysis of Charge Photogeneration as a Key Determinant of Photocurrent Density in Polymer: Fullerene Solar Cells. Adv. Mater. 2010, 22, 5287-5291.

(16) Cha, H.; Tan, C.-H.; Wu, J.; Dong, Y.; Zhang, W.; Chen, H.; Rajaram, S.; Narayan, K. S.; McCulloch, I.; Durrant, J. R. An Analysis of the Factors Determining the Efficiency of Photocurrent Generation in Polymer:Nonfullerene Acceptor Solar Cells. Adv. Energy Mater. 2018, 8, 1801537.

(17) Fallon, K. J.; Wijeyasinghe, N.; Leventis, A.; Marin-Beloqui, J. M.; Al-Hashimi, M.; Clarke, T.; Anthopoulos, T. D.; Bronstein, H. Tyrian Purple: An Ancient Natural Dye for Cross-Conjugated NType Charge Transport. Submitted for publication.

(18) Yoshida, H. Low-Energy Inverse Photoemission Study on the Electron Affinities of Fullerene Derivatives for Organic Photovoltaic Cells. J. Phys. Chem. C 2014, 118, 24377-24382.

(19) Davis, R. J.; Lloyd, M. T.; Ferreira, S. R.; Bruzek, M. J.; Watkins, S. E.; Lindell, L.; Sehati, P.; Fahlman, M.; Anthony, J. E.; Hsu, J. W. P. Determination of Energy Level Alignment at Interfaces of Hybrid and Organic Solar Cells under Ambient Environment. J. Mater. Chem. 2011, 21, 1721-1729.

(20) Fallon, K. J.; Wijeyasinghe, N.; Manley, E. F.; Dimitrov, S. D.; Yousaf, S. A.; Ashraf, R. S.; Duffy, W.; Guilbert, A. Y.; Freeman, D. M. E.; Al-Hashimi, M.; et al. Indolo-Naphthyridine-6,13-Dione Thiophene Building Block for Conjugated Polymer Electronics: Molecular Origin of Ultrahigh n-Type Mobility. Chem. Mater. 2016, 28, 83668378. 
(21) Hood, S. N.; Kassal, I. Entropy and Disorder Enable Charge Separation in Organic Solar Cells. J. Phys. Chem. Lett. 2016, 7, 44954500.

(22) Qian, D.; Zheng, Z.; Yao, H.; Tress, W.; Hopper, T. R.; Chen, S.; Li, S.; Liu, J.; Chen, S.; Zhang, J.; et al. Design Rules for Minimizing Voltage Losses in High-Efficiency Organic Solar Cells. Nat. Mater. 2018, 17, 703-709.

(23) Lawson, D. R; Feldhiem, D. L.; Foss, C. A.; Dorhout, P. K.; Elliott, C. M.; Martin, C. R.; Parkinson, B. Near-IR Absorption Spectra for the $\mathrm{C}_{70}$ Fullerene Anions. J. Phys. Chem. 1992, 96, 71757177.

(24) Yamamoto, S.; Guo, J.; Ohkita, H.; Ito, S. Formation of Methanofullerene Cation in Bulk Heterojunction Polymer Solar Cells Studied by Transient Absorption Spectroscopy. Adv. Funct. Mater. 2008, 18, 2555-2562.

(25) Guo, J.; Ohkita, H.; Benten, H.; Ito, S. Near-IR Femtosecond Transient Absorption Spectroscopy of Ultrafast Polaron and Triplet Exciton Formation in Polythiophene Films with Different Regioregularities. J. Am. Chem. Soc. 2009, 131, 16869-16880.

(26) Piris, J.; Dykstra, T. E.; Bakulin, A. A.; van Loosdrecht, P. H. M.; Knulst, W.; Trinh, M. T.; Schins, J. M.; Siebbeles, L. D. A. Photogeneration and Ultrafast Dynamics of Excitons and Charges in P3HT/PCBM Blends. J. Phys. Chem. C 2009, 113, 14500-14506.

(27) Nelson, J. Diffusion-Limited Recombination in PolymerFullerene Blends and Its Influence on Photocurrent Collection. Phys. Rev. B: Condens. Matter Mater. Phys. 2003, 67, 155209.

(28) Clarke, T. M.; Jamieson, F. C.; Durrant, J. R. Transient Absorption Studies of Bimolecular Recombination Dynamics in Polythiophene/Fullerene Blend Films. J. Phys. Chem. C 2009, 113, 20934-20941.

(29) Clarke, T.; Ballantyne, A.; Jamieson, F.; Brabec, C.; Nelson, J.; Durrant, J. Transient Absorption Spectroscopy of Charge Photogeneration Yields and Lifetimes in a Low Bandgap Polymer/ Fullerenefilm. Chem. Commun. 2008, 7345, 89-91.

(30) Liu, T.; Huo, L.; Chandrabose, S.; Chen, K.; Han, G.; Qi, F.; Meng, X.; Xie, D.; Ma, W.; Yi, Y.; et al. Optimized Fibril Network Morphology by Precise Side-Chain Engineering to Achieve HighPerformance Bulk-Heterojunction Organic Solar Cells. Adv. Mater. 2018, 30, 1707353.

(31) Yang, X.; Loos, J.; Veenstra, S. C.; Verhees, W. J. H.; Wienk, M. M.; Kroon, J. M.; Michels, M. A. J.; Janssen, R. A. J. Nanoscale Morphology of High-Performance Polymer Solar Cells. Nano Lett. 2005, 5, 579-583.

(32) Tautz, R.; Da Como, E.; Limmer, T.; Feldmann, J.; Egelhaaf, H.-J.; von Hauff, E.; Lemaur, V.; Beljonne, D.; Yilmaz, S.; Dumsch, I.; Allard, S.; Scherf, U. Structural Correlations in the Generation of Polaron Pairs in Low-Bandgap Polymers for Photovoltaics. Nat. Commun. 2012, 3, 970.

(33) Clarke, T. M.; Lungenschmied, C.; Peet, J.; Drolet, N.; Sunahara, K.; Furube, A.; Mozer, A. J. Photodegradation in Encapsulated Silole-Based Polymer: PCBM Solar Cells Investigated Using Transient Absorption Spectroscopy and Charge Extraction Measurements. Adv. Energy Mater. 2013, 3, 1473-1483.

(34) Xu, H.; Jiang, Y.; Li, J.; Ong, B. S.; Shuai, Z.; Xu, J.; Zhao, N. Spectroscopic Study of Electron and Hole Polarons in a HighMobility Donor-Acceptor Conjugated Copolymer. J. Phys. Chem. C 2013, 117, 6835-6841.

(35) Guo, J.; Ohkita, H.; Yokoya, S.; Benten, H.; Ito, S. Bimodal Polarons and Hole Transport in Poly(3-hexylthiophene):Fullerene Blend Films. J. Am. Chem. Soc. 2010, 132, 9631-9637.

(36) Arkhipov, V. I.; Heremans, P.; Bässler, H. Why Is Exciton Dissociation so Efficient at the Interface between a Conjugated Polymer and an Electron Acceptor? Appl. Phys. Lett. 2003, 82, 46054607. 